\title{
A comprehensive introduction to the genetic basis of non-syndromic hearing loss in the Saudi Arabian population
}

\author{
Faiqa Imtiaz ${ }^{\text {* }}$, Khalid Taibah², Khushnooda Ramzan', Ghada Bin-Khamis ${ }^{3}$, Shelley Kennedy ${ }^{4}$, Bashayer Al-Mubarak, \\ Daniah Trabzuni ${ }^{1}$, Rabab Allam ${ }^{1}$, Abeer Al-Mostafa', Sameera Sogaty ${ }^{5}$, Abdulmoneem H Al-Shaikh ${ }^{6}$, \\ Saeed S Bamukhayyar, Brian F Meyer ${ }^{1}$ and Mohammed Al-Owain ${ }^{8,9}$
}

\begin{abstract}
Background: Hearing loss is a clinically and genetically heterogeneous disorder. Mutations in the DFNB1 locus have been reported to be the most common cause of autosomal recessive non-syndromic hearing loss worldwide. Apart from DFNB1, many other loci and their underlying genes have also been identified and the basis of our study was to provide a comprehensive introduction to the delineation of the molecular basis of non-syndromic hearing loss in the Saudi Arabian population. This was performed by screening DFNB1 and to initiate prioritized linkage analysis or homozygosity mapping for a pilot number of families in which DFNB1 has been excluded.

Methods: Individuals from 130 families of Saudi Arabian tribal origin diagnosed with an autosomal recessive nonsyndromic sensorineural hearing loss were screened for mutations at the DFNB1 locus by direct sequencing. If negative, genome wide linkage analysis or homozygosity mapping were performed using Affymetrix GeneChip ${ }^{\circledR}$ Human Mapping 250K/6.0 Arrays to identify regions containing any known-deafness causing genes that were subsequently sequenced.

Results: Our results strongly indicate that DFNB1 only accounts for $3 \%$ of non-syndromic hearing loss in the Saudi Arabian population of ethnic ancestry. Prioritized linkage analysis or homozygosity mapping in five separate families established that their hearing loss was caused by five different known-deafness causing genes thus confirming the genetic heterogeneity of this disorder in the kingdom.

Conclusion: The overall results of this study are highly suggestive that underlying molecular basis of autosomal recessive non-syndromic deafness in Saudi Arabia is very genetically heterogeneous. In addition, we report that the preliminary results indicate that there does not seem to be any common or more prevalent loci, genes or mutations in patients with autosomal recessive non-syndromic hearing loss in patients of Saudi Arabian tribal origin.
\end{abstract}

\section{Background}

Deafness, the inability to hear, is the most common sensory deficit in human populations with both genetic and environmental etiologies. It is estimated that it affects 1 in 1000 child births of which approximately $60 \%$ cases are attributed to genetic factors [1]. Hearing impairment is clinically and genetically heterogeneous. Impaired

\footnotetext{
* Correspondence: fahmad@kfshrc.edu.sa

'Department of Genetics, King Faisal Specialist Hospital \& Research Centre,

PO Box 3354, Riyadh 11211, Saudi Arabia

Full list of author information is available at the end of the article
}

auditory function can be the only clinical manifestation (non-syndromic forms of deafness) or can be associated with other symptoms or anomalies (syndromic forms of deafness). It has been estimated that at least 300 human protein-coding genes are involved in the hearing process [2]. During the last decade, many deafness loci and the underlying genes have been identified at a rapid rate.

The main pattern of inheritance in severe childhood deafness is autosomal recessive (over $75 \%$ ) while autosomal dominant (12-24\%), X-linked (1-3\%) and mitochondrial is also involved [3]. In general, recessive deafness is

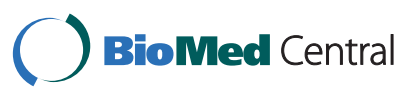


more likely to be more severe than dominant deafness because it is generally profound, prelingual and fully penetrant whereas dominant deafness is often progressive, post lingual and is frequently observed clinically as unilateral or mild bilateral deafness [4]. In addition, recessively inherited diseases are more prevalent in populations where consanguineous marriages are common, like in the kingdom of Saudi Arabia. The same effect is observed for recessively inherited cases of deafness.

Autosomal recessive non-syndromic hearing loss (ARNSHL) is the most frequent cause of hereditary deafness and often exhibits the most severe hearing phenotype. Presently, 85 recessive deafness (DFNB) loci have been registered and 35 of the corresponding genes have been identified as documented on the Hereditary Hearing Loss Homepage http://hereditaryhearingloss.org. Interestingly, even though a substantial number of genes are known to cause ARNSHL, mutations at the first identified DFNB1 locus, account for up to $50 \%$ of all cases with this diagnosis in various populations. DFNB1 contains the GJB2 and GJB6 genes that respectively code for connexin $26(\mathrm{C} \times 26)$ and connexin $30(\mathrm{C} \times 30)$, which are gap junction binding proteins, most abundantly expressed in the cochlea. Although over 100 mutations have been reported in GJB2 (The Connexin-deafness homepage: http://davinci.crg.es/ deafness), a single mutation, c.35delG, is the most common cause of ARNSHL and can account for up to $85 \%$ of DFNB1 in various populations [5]. DFNB1 also harbors the second most common ARNSHL causing mutation, a $309 \mathrm{~kb}$ deletion of GJB6, often found in trans with mutations in GJB2, giving rise to a digenic inheritance of this form of hereditary hearing loss.

In view of the extensive studies conducted worldwide, the major objective of this study was to begin to comprehensively delineate the genetic basis of ARNSHL in individuals of Saudi Arabian origin. To achieve this objective, individuals segregating with severe to profound, nonsyndromic congenital deafness with an autosomal recessive mode of inheritance were identified from different cities of Saudi Arabia and initially screened for mutations encompassing the DFNB1 locus.

The second aim was to initiate prioritized linkage analysis (in families of three or more affected individuals) and homozygosity mapping (in families with two affected sib-pair individuals) of other known deafness loci for families in which DFNB1 has been excluded. This further bi-directional approach was necessary due to the vast heterogeneity and complexity of the number of genes known and unknown causing hereditary deafness. In this study, using SNP-based genotyping arrays, candidate genes were selected and screened in the chromosomal region that generated the highest LOD score (multipoint linkage analysis) or in the largest region of homozygosity shared by the affected sib-pairs that was not seen in the parents or unaffected siblings if applicable. This genomewide homozygosity mapping analysis approach was used in this study in particular, as it assumes that individuals affected with an autosomal recessive disease, born from parents of a consanguineous marriage are very likely to be homozygous for the pathogenic mutation and for a substantial number of SNPs surrounding it [6].

The overall results of this study are highly suggestive that underlying molecular basis of autosomal recessive non-syndromic deafness in Saudi Arabia is very genetically heterogeneous. In addition, we report that from the results of our pilot study there does not seem to be any common or more prevalent loci, genes or mutations in patients with ARNSHL originating from the kingdom.

\section{Methods \\ Subjects}

All of the individuals (patients and family members) who participated in this study provided an approved informed consent form, which adhered to institutional (King Faisal Specialist Hospital; RAC\# 2040039) and international guidelines as outlined by the Declaration of Helsinki.

\section{Clinical evaluation}

Detailed medical histories were obtained for all of the enrolled affected individuals regarding time and age of onset of hearing loss (pre-lingual, post-lingual), severity of hearing loss (mild, moderate or profound) and in order to exclude syndromic abnormalities, environmental causes for hearing loss and an autosomal dominant inheritance pattern of hearing loss. Pure tone audiometry tests for air and bone conduction were performed on all affected individuals. Evaluation of vestibular and ocular function was performed to exclude suspected forms of syndromic deafness.

\section{Sample Collection and DNA Extraction}

Whole blood samples $(10 \mathrm{ml})$ for molecular genetic analysis were obtained from affected patients, their parents and unaffected siblings (if available). Genomic extraction of DNA was performed using the standard salting-out method [7].

\section{Mutation Detection in GJB2 and GJB6}

Mutation screening by direct dideoxy chain-termination sequencing of the entire coding region of PCR generated amplicons (using standard PCR conditions) was performed for both the GJB2 and GJB6 genes in all affected individuals in the study using an ABI Prism Big Dye Terminator v3.1 Cycle Sequencing Kit following the manufacturer's instructions and processed on a MegaBACE 1000 DNA Analysis System (Molecular Dynamics; 
Sunnyvale, CA, USA). Sequence analysis was performed using the SeqMan 6.1 module of the Lasergene (DNA Star Inc. WI, USA) software package, then compared to the reference GenBank sequence (accession number: GJB2; NM_004004 and GJB6; NM_001110219). Numbering commenced with the A of the ATG initiation codon as +1 .

\section{Linkage analysis}

SNP-based genotyping was performed using the Affymetrix GeneChip ${ }^{\circledR}$ Human Mapping $250 \mathrm{~K}$ and 6.0 Arrays (Affymetrix, Santa Clara, CA, USA) as per the user's manual. The genotypes of SNPs were called using Affymetrix GCOS 1.4 software, which generated an overall average SNP call rate of $97 \%$ and were further sorted per chromosome and by physical position. The Allegro module of the Easy Linkage software package was used to calculate multipoint logarithm of odds (LOD) scores, with the parameters that assume a disease model with an autosomal-recessive mode of inheritance with $100 \%$ penetrance and a disease allele frequency of 0.0001 .

The resulting multipoint linkage analysis of the affected patients, their unaffected siblings, and their parents which resulted in the highest maximum LOD score was investigated. Any known deafness causing gene(s) located in this interval were assumed the likely diseasecausing candidate and the patients were screened by direct sequencing of the coding sequence for the particular gene(s).

\section{Homozygosity mapping}

The resulting SNP-based genotyping data (as in Linkage Analysis) was analyzed to detect regions of homozygosity using CNAG 3.0 and GTConsole Version 3.0.1 (Affymetrix, Santa Clara, CA, USA) software packages. Conventionally, regions of homozygosity are defined as fragments where SNPs are homozygous for a stretch of consecutive alleles in affected individuals and heterozygous or homozygous for the other allele in unaffected members of the same family. As previously reported by researchers using this approach [8], it is advantageous to identify and initially screen candidate genes present in the largest region of homozygosity shared between affected family members, however this was not true in all families that were analyzed in this manner.

\section{Mutation Detection in MYO15A, TMPRSS3, TRIC, LHFPL5, TMC1}

After linkage and homozygosity mapping analysis, genomic DNA from relevant individuals was amplified using standard PCR conditions with intronic primers that were designed to flank (50-100 bp) each of coding exons of MYO15A, TMPRSS3, TRIC, LHFPL5 and TMC1 as mentioned previously (primer sequences and conditions are available on request).

\section{RT-PCR}

To determine the effect of the novel splice-acceptor site mutation (IVS12-1 g>c) in family KT1 with respect to mRNA splicing, a fragment comprising exon 11 and 12 of TMPRSS3 was amplified using RT-PCR with mRNA isolated from peripheral blood lymphocytes. Preparation of the cDNA was carried out using the iScript ${ }^{\mathrm{TM}} \mathrm{cDNA}$ synthesis kit and random primers (Applied Biosystems, Carlsbad, CA). One microgram of total RNA from the patient and a normal control was reverse transcribed using an aliquot of a reverse transcription reaction containing $1 \times$ PCR buffer, $2.5 \mathrm{mM} \mathrm{MgCl}_{2}, 0.2 \mathrm{mM}$ of each $\mathrm{dNTP}$, and 0.5 U AMV reverse transcriptase (Roche Diagnostics GmbH, Mannheim, Germany). $10 \mu \mathrm{l}$ of cDNA was amplified by PCR using the $5^{\prime}$ primer (TCC AAC AAG ATC TGC AAC CA) and the 3' primer (ATC CAG TCC AGG AAG GAG GT) using standard conditions. PCR products were then evaluated on $2 \%$ agarose gel and were purified using the QIAquick PCR Purification Kit according to the manufacturer's instructions (Qiagen, Germantown, MD) and then sequenced as described above.

\section{Results}

GJB2 and GJB6 were screened from affected individuals with pre-lingual severe to profound ARNSHL from a total of 130 families, which included both multiple affected and sporadic cases. DFNB1 mutations were identified in only 4 of the 130 families. Patients from two of the families were homoallelic for the common c.35delG mutation in GJB2. One patient was homozygous for the $\mathrm{R} 32 \mathrm{H}$ mutation and the fourth patient was homozygous for W77R, both of which have been previously reported in GJB2 (Table 1). No other mutations have been found in GJB2 or GJB6 (including the 309-kb deletion) in this largest cohort of patients from this population. Therefore, our results show that mutations in DFNB1 account for only 3\% of ARNSHL in patients of Saudi Arabian tribal ethnicity.

\section{Linkage analysis and homozygosity mapping in $\mathbf{5}$ Families with ARNSHL}

The exclusion of DFNB1 as a major cause of ARNSHL allowed us to accelerate our efforts with respect to linkage analysis and homozygosity mapping. 70 of the 130 families that were enrolled in the study had 2 or more affected individuals, the remaining being made up of sporadic cases. Initially as a pilot study, patients from five families were selected for analysis on the basis that the parents confirmed consanguineous marriages and that the non-syndromic hearing loss was consistent with a 
Table 1 Summary of mutations in various known deafness genes identified in ARNSHL patients from Saudi Arabia

\begin{tabular}{|c|c|c|c|c|c|c|}
\hline $\begin{array}{l}\text { FAMILY/ } \\
\text { SIBSHIPS }\end{array}$ & MODE OF ANALYSIS & $\begin{array}{l}\text { REGION OF } \\
\text { INTEREST }\end{array}$ & $\begin{array}{l}\text { GENE OF } \\
\text { INTEREST }\end{array}$ & $\begin{array}{l}\text { NUCLEOTIDE } \\
\text { CHANGE }\end{array}$ & $\begin{array}{l}\text { PROTEIN } \\
\text { CHANGE }\end{array}$ & REFERENCE \\
\hline NSHD28/69 & DFNB1 SCREENING & NA & GJB2 & c.35delG & Frameshift & [10] \\
\hline NSHD74 & DFNB1 SCREENING & NA & GJB2 & $95 \mathrm{G}>\mathrm{A}$ & p.R32H & {$[11]$} \\
\hline KT7 & DFNB1 SCREENING & NA & GJB2 & c. $229 \mathrm{~T}>\mathrm{C}$ & p.W77R & [12] \\
\hline SS16 & $\begin{array}{c}\text { Linkage } \\
\text { (LOD SCORE) }\end{array}$ & Chr 17p12-q11.2 & MYO15A & c.1047C >A & p.Y349X & This study \\
\hline KT1 & $\begin{array}{c}\text { Linkage } \\
\text { (LOD SCORE) }\end{array}$ & Chr 21q22.2-q22.3 & TMPRSS3 & IVS12-1 g>C & Splicing error & This study \\
\hline TA12 & $\begin{array}{c}\text { Homozygosity (GT } \\
\text { console) }\end{array}$ & Chr 5q11.2-q13.3 & TRIC & c. $1498 C>T$ & p.R500X & [13] \\
\hline SS3 & $\begin{array}{c}\text { Homozygosity (GT } \\
\text { console) }\end{array}$ & Chr 6p24.3-p12.3 & LHFPL5 & c. $1 \mathrm{~A}>\mathrm{G}$ & p.M1V & {$[8]$} \\
\hline SS17 & Homozygosity (CNAG) & Chr 9p13.3-q21.13 & TMC1 & c. $100 C>T$ & p.R34X & [14] \\
\hline
\end{tabular}

recessive mode of inheritance. Two families of 3 affected individuals were subjected to LOD score calculation by linkage analysis and the remaining 3 families with two affected individuals were analyzed by homozygosity mapping (Figure 1). Multipoint linkage analysis of the first family generated a LOD score of 2.5 on chromosome 17p12-p11.2 in which $M Y O 15 A$ was selected as a candidate within a $6.0 \mathrm{Mb}$ linkage interval. Similar analysis on the second family resulted in a maximum LOD score of 2.7 (Figure 1) and identified a disease locus on chromosome 21q22.3, which spanned approximately $1.5 \mathrm{Mb}$. TMPRSS3 was selected as a potential candidate in this region. Homozygosity mapping analysis in the remaining 3 sibships generated multiple regions of homozygosity that was shared only among affected individuals. But as previously discussed, any known DFNB genes that were located in the longest stretch of homozygosity were chosen for sequencing in the respective family. On this basis, TRIC, LHFPL5, and TMC1 were selected to be directly sequenced from the genomic DNA

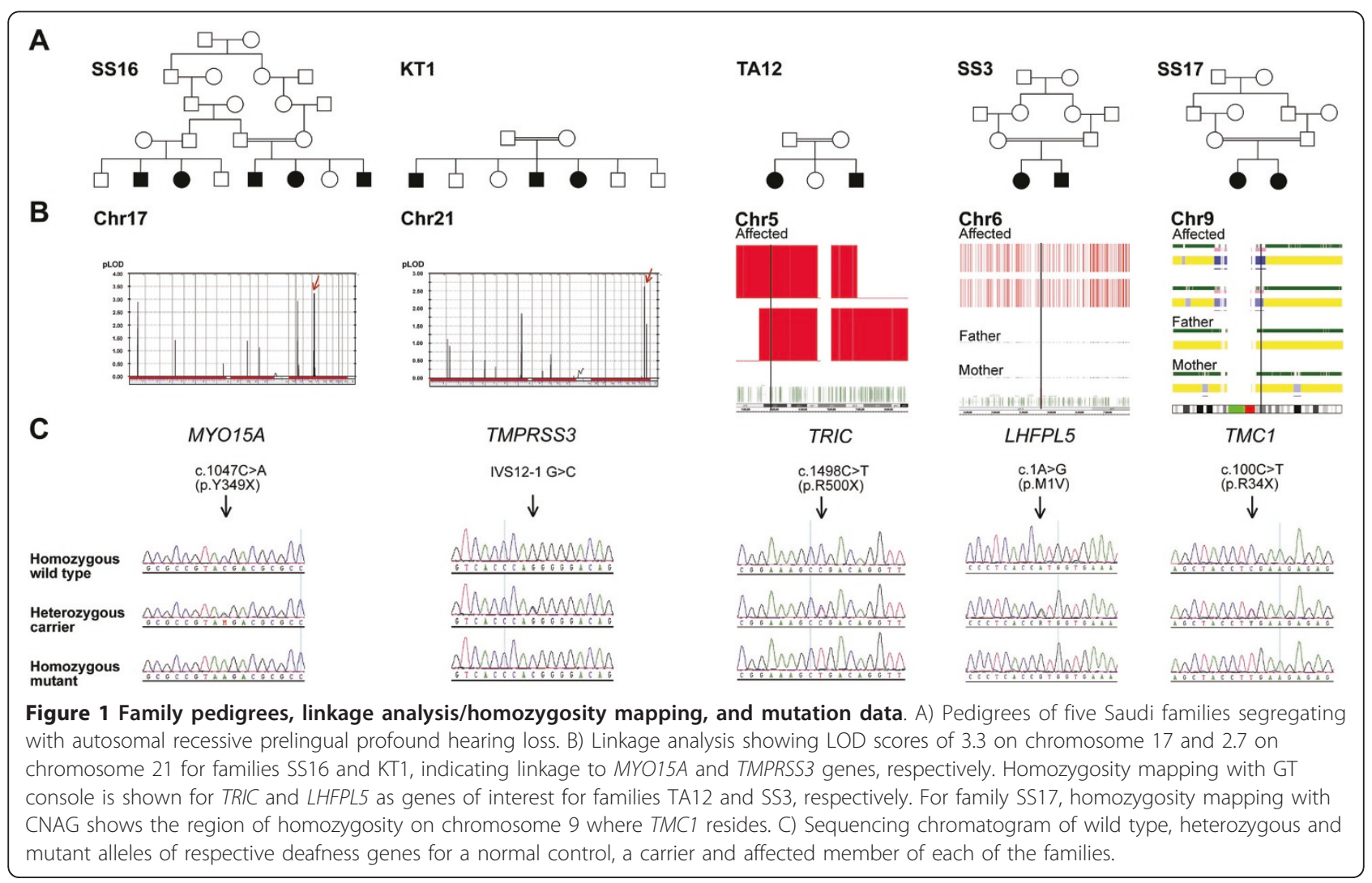


of all members in the particular family that affected patients were homozygous for the interval containing the applicable gene.

\section{Mutation Screening in MYO15A, TMPRSS3, TRIC, LHFPL5, TMC1}

Direct sequencing in both the forward and reverse directions of each candidate gene that the respective affected siblings linked to, revealed the presence of homozygous mutations in all of the five genes (Figure 1). The mutations segregated with the disease phenotype where applicable, i.e. parents were heterozygous carriers for the mutation and unaffected siblings were either heterozygous carriers or wild-type normal. Two of the five mutations found were novel (Table 1). Neither of the novel mutations were found in 300 ethnically matched normal controls, which indicated that these variants were not population-based polymorphisms.

RT-PCR products were utilized to establish the effect of the novel splice-acceptor site mutation (IVS12-1 g>c) in the family KT1 with respect to mRNA splicing in TMPRSS3. Sequencing results provided the evidence that the patients which are homozygous for a novel intronic splice-acceptor site mutation shows the activation of cryptic splice site 8 bp downstream in the exon 12 leading to a frameshift and incorporation of faulty amino acids and hence confirms that this mutation does indeed affect normal splicing and had a major deleterious impact on the structure of the TMPRSS3 mRNA (Figure 2).

\section{Discussion}

Our results strongly indicate that DFNB1 only accounts for 3\% of ARNSHL in the Saudi population (Table 1). This percentage is significantly lower than a recent study by Al-Qahtani et al [9] who reported that the total mutations in GJB2 account for $15.59 \%$ in their cohort of patients from the western province of Saudi Arabia. However, this number is most likely elevated compared to our results as they reported GJB2 mutation findings on patients of different ethnic origins, which they also suggest may be a consequence of migration and settlement in the geographical area of where their study was conducted. All of the patients in this study were of Saudi Arabian tribal origin and therefore, we believe that $3 \%$ is a more accurate estimation of the contribution of DFNB1 in ARNSHL in individuals ethnically originating from the Kingdom.

Three of the five mutations identified by direct sequencing of known deafness genes in the candidate regions identified by linkage analysis/homozygosity mapping in 5 families in which DFNB1 had been excluded have been reported previously (Table 1 ). The novel nonsense mutation detected in MYO15A in family SS16
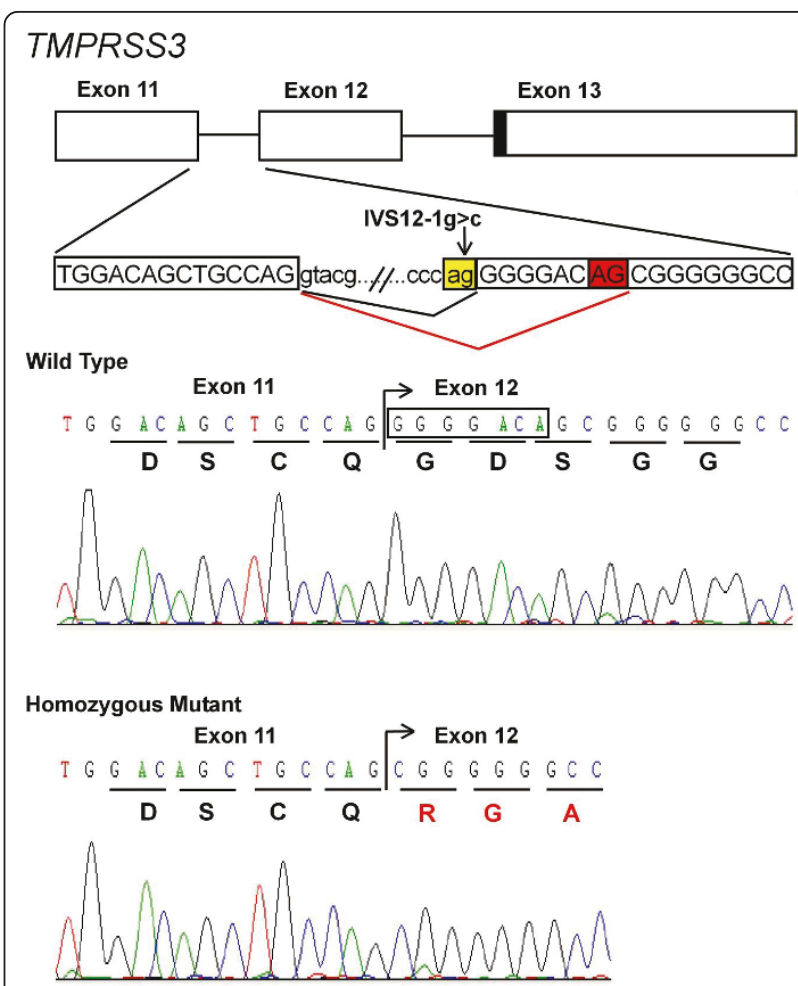

Figure 2 Schematic presentation for the genomic localization and the sequence of splice site mutation. The IVS12-1 $\mathrm{g}>\mathrm{C}$ mutation (indicated by an arrow) creates a $5^{\prime}$ cryptic acceptor site (AG boxed in red) 8 bp downstream in exon 12 instead of a normal acceptor site (ag boxed in yellow). Sequencing of the mutant transcript shows that cryptic splice site leads to a frameshift and incorporation of faulty amino acids as compared to the wild type.

results in the premature truncation at amino acid position 349 located in the first domain (N-terminal extension) of the myosin XVa protein, which is normally comprised of 3530 amino acids. The second novel mutation, a splice-acceptor site disruption (IVS12-1 g>c) in the TMPRSS3 gene in family KT1 was confirmed by RT-PCR to cause abnormal splicing by activation of a cryptic splice site resulting in a frameshift and incorporation of faulty amino acids in this serine protease.

Consanguineous families are a powerful resource for genetic linkage studies of recessively inherited hearing impairment. So far, family based analyses have proven that this methodology is successful and the results have shown that the hearing loss in five separate families was caused by five different genes (Table 1), thus tentatively confirming the already well-established finding of genetic heterogeneity in ARNSHL worldwide is in fact analogous in the Saudi population. From the combined approach and data of the current study, it is not possible to exclude the possibility of more prevalent ARNSHLcausing genes in this population that have not yet been identified. However, it is pertinent to highlight that this 
is a pilot study and the findings discussed here will be validated by our future direction once results are obtained from further similar family-based analyses, in addition to whole exome/genome sequencing of our large number of families with a single affected proband that are not amenable to analysis using the same linkage and homozygosity mapping methodology detailed in this preliminary report.

\section{Conclusion}

In conclusion, DFNB1 is a very minor cause of ARNSHL in individuals of Saudi Arabian tribal origin. By using the effective bi-directional approach of linkage analysis and homozygosity mapping that we have initiated, we will be able to identify the most common forms of hereditary hearing loss, their incidence and distribution in the Saudi population. The benefit of this study will hopefully provide the foundation for knowledge and awareness through screening of carrier status and genetic counselling, thereby having a major impact upon early intervention for and prevention of hereditary hearing loss.

\section{Acknowledgements}

This work was funded and supported in part by King Faisal Specialist Hospital (RAC\#2040039), the Prince Salman Centre for Disability Research through a charitable donation from Sheikh Nasser Al-Mutawa (PSCDR \#04IN-0005-04-EP-1) and the Dubai Harvard Foundation for Medical Research for a Research Fellowship awarded to Dr. Faiqa Imtiaz.

\section{Author details}

${ }^{1}$ Department of Genetics, King Faisal Specialist Hospital \& Research Centre, PO Box 3354, Riyadh 11211, Saudi Arabia. 'ENT Medical Centre, PO 340507, Riyadh 11333, Saudi Arabia. ${ }^{3}$ Department of Otololaryngology, King Faisal Specialist Hospital \& Research Centre, PO Box 3354, Riyadh 11211, Saudi Arabia. ${ }^{4}$ Ontario Newborn Screening Program, Children's Hospital of Eastern Ontario 401 Smyth Road, Room 3127A Ottawa, Ontario K1H 8L1, Canada. ${ }^{5}$ Medical Genetics Unit, King Fahad Hospital, PO Box 8488, Jeddah 21196, Saudi Arabia. ${ }^{6}$ ENT Department, King Fahad Hospital, PO Box 8488, Jeddah 21196, Saudi Arabia. ${ }^{7}$ Audio-Vestibular Medicine Unit, King Fahd Hospital, PO Box 8488, Jeddah 21196, Saudi Arabia. ${ }^{8}$ Department of Medical Genetics, King Faisal Specialist Hospital \& Research Centre, PO Box 3354, Riyadh 11211, Saudi Arabia. ${ }^{9}$ College of Medicine, Al-Faisal University, PO Box 50927, Riyadh 11533, Saudi Arabia.

\section{Authors' contributions}

FI was primarily responsible for the design, molecular genetic studies, data interpretation, drafting and finalizing the manuscript; KT and MO were primarily responsible for clinical support, clinical evaluation and sample collection; MO also participated in editing of the manuscript; KR participated in molecular genetic studies and result databasing and formatting, GBK was responsible for audiological evaluation; BAM, DT, RA and AM assisted in carrying out molecular genetic studies; participated in methodology; SS, SSB and AHS contributed with sample collection, ENT and genetic evaluation; BFM participated in design, data interpretation and final editing of the manuscript. All authors have read and approved the final manuscript.

\section{Competing interests}

The authors declare that they have no competing interests.

\section{References}

1. Marazita ML, Ploughman LM, Rawlings B, Remington E, Arnos KS, Nance WE: Genetic epidemiological studies of early-onset deafness in the U.S. school-age population. Am J Med Genet 1993, 46:486-491.

2. Friedman RA, Bykhovskaya Y, Sue CM, DiMauro S, Bradley R, FallisCunningham R, Paradies N, Pensak ML, Smith RJ, Groden J, Li XC, FischelGhodsian N: Maternally inherited nonsyndromic hearing loss. Am J Med Genet 1999, 84:369-372.

3. Morton NE: Genetic epidemiology of hearing impairment. Ann N Y Acad Sci 1991, 630:16-31.

4. Bitner-Glindzicz M: Hereditary deafness and phenotyping in humans. $\mathrm{Br}$ Med Bull 2002, 63:73-94.

5. Kenneson A, Van Naarden Braun K, Boyle C: GJB2 (connexin 26) variants and nonsyndromic sensorineural hearing loss: a HuGE review. Genet Med 2002, 4:258-274.

6. Woods CG, Cox J, Springell K, Hampshire DJ, Mohamed MD, McKibbin M, Stern R, Raymond FL, Sandford R, Malik Sharif S, Karbani G, Ahmed M, Bond J, Clayton D, Inglehearn CF: Quantification of homozygosity in consanguineous individuals with autosomal recessive disease. Am J Hum Genet 2006, 78:889-896.

7. Miller SA, Dykes DD, Polesky HF: A simple salting out procedure for extracting DNA from human nucleated cells. Nucleic Acids Res 1988, 16:1215.

8. Shahin H, Walsh T, Rayyan AA, Lee MK, Higgins J, Dickel D, Lewis K, Thompson J, Baker C, Nord AS, Stray S, Gurwitz D, Avraham KB, King MC, Kanaan M: Five novel loci for inherited hearing loss mapped by SNPbased homozygosity profiles in Palestinian families. Eur J Hum Genet 2010, 18:407-413.

9. Al-Qahtani MH, Baghlab I, Chaudhary AG, Abuzenadah AM, Bamanie A, Daghistani KJ, Safieh M, Fida L, Dallol A: Spectrum of GJB2 mutations in a cohort of nonsyndromic hearing loss cases from the Kingdom of Saudi Arabia. Genet Test Mol Biomarkers 2010, 14:79-83.

10. Zelante L, Gasparini P, Estivill X, Melchionda S, D'Agruma L, Govea N, Mila M, Monica MD, Lutfi J, Shohat M, Mansfield E, Delgrosso K, Rappaport E, Surrey S, Fortina P: Connexin26 mutations associated with the most common form of non-syndromic neurosensory autosomal recessive deafness (DFNB1) in Mediterraneans. Hum Mol Genet 1997, 6:1605-1609.

11. Marlin S, Garabedian EN, Roger G, Moatti L, Matha N, Lewin P, Petit C, Denoyelle F: Connexin 26 gene mutations in congenitally deaf children: pitfalls for genetic counseling. Arch Otolaryngol Head Neck Surg 2001, 127:927-933

12. Carrasquillo MM, Zlotogora J, Barges S, Chakravarti A: Two different connexin 26 mutations in an inbred kindred segregating non-syndromic recessive deafness: implications for genetic studies in isolated populations. Hum Mol Genet 1997, 6:2163-2172.

13. Riazuddin S, Ahmed ZM, Fanning AS, Lagziel A, Kitajiri S, Ramzan K, Khan SN, Chattaraj P, Friedman PL, Anderson JM, Belyantseva IA, Forge A, Friedman TB: Tricellulin is a tight-junction protein necessary for hearing. Am J Hum Genet 2006, 79:1040-1051.

14. Kurima K, Peters LM, Yang Y, Riazuddin S, Ahmed ZM, Naz S, Arnaud D, Drury S, Mo J, Makishima T, Ghosh M, Menon PS, Deshmukh D, Oddoux C, Ostrer H, Khan S, Deininger PL, Hampton LL, Sullivan SL, Battey JF, Keats BJ, Wilcox ER, Friedman TB, Griffith AJ: Dominant and recessive deafness caused by mutations of a novel gene, TMC1, required for cochlear haircell function. Nat Genet 2002, 30:277-284.

\section{Pre-publication history}

The pre-publication history for this paper can be accessed here: http://www.biomedcentral.com/1471-2350/12/91/prepub

doi:10.1186/1471-2350-12-91

Cite this article as: Imtiaz et al:: A comprehensive introduction to the genetic basis of non-syndromic hearing loss in the Saudi Arabian population. BMC Medical Genetics 2011 12:91. 